\title{
Efeito da imersão em água destilada sobre os parâmetros reprodutivos de teleóginas de Rhipicephalus (Boophilus) microplus
}

\section{Water immersion effect on reproductive parameters of Rhipicephalus (Boophilus) microplus ticks}

\author{
Fernanda Carlini Cunha dos Santos ${ }^{1 *}$; Fernanda Silveira Flores Vogel ${ }^{2}$; \\ Silvia Gonzalez Monteiro ${ }^{3}$; Victor Fernando Buttow Roll ${ }^{4}$
}

\begin{abstract}
Resumo
O carrapato Rhipicephalus (Boophilus) microplus é um parasita de destaque na pecuária brasileira devido às perdas econômicas causadas ao rebanho bovino. Este parasito apresenta duas fases de vida, uma fase parasitária que ocorre no corpo do hospedeiro e uma fase de vida livre que ocorre no solo e vegetação. O acúmulo de água no solo influencia na biologia dos ixodídeos, além de outros fatores fundamentais como a temperatura e a umidade relativa do ar. Este experimento teve a intenção de investigar a ação deletéria da imersão em água, em condições controladas, de fêmeas de $R$. microplus. Para isso, as teleóginas permaneceram imersas em água por $0,2,4,6,12,18,24,30,36,42,48$, $60,72,84,96,108$ e 120 horas conforme tratamento, sendo posteriormente colocadas em uma estufa climatizada para verificação da postura de ovos e eclosão das larvas. Foi observado que em um período de até $30 \mathrm{~h}$ de imersão, as teleóginas realizaram postura, no entanto mais de $50 \%$ ovos não eclodiram. Após $48 \mathrm{~h}$, foi observado a postura em mais de $50 \%$ das fêmeas e a taxa de eclosão larval foi inferior a $31 \%$. A partir de $84 \mathrm{~h}$ não houve mais eclosão larval e o tratamento atingiu $100 \%$ de eficiência. Com base nos resultados obtidos, conclui-se que a imersão em água por um período superior a 2 horas possui efeito negativo nos parâmetros reprodutivos do carrapato bovino, através de alterações na postura e na eclosão larval.

Palavras-chave: Água destilada, eclosão de larvas, imersão, Rhipicephalus (Boophilus) microplus
\end{abstract}

\begin{abstract}
The tick Rhipicephalus (Boophilus) microplus is an important parasite in the cattle raising field due to the economic losses it causes to Brazilian herds. This parasite has two stages of life, a parasitic stage that takes place in the host body and a free-living stage that takes place on soil and vegetation. The water accumulation on the soil has a strong influence in the biology of the Ixodidae, as well as other factors such as temperature and relative humidity. The aim of this experiment was to evaluate the possible deleterious effect of water immersion in controlled conditions of $R$. microplus females. For that, the teleogines were immersed in water for $0,2,4,6,12,18,24,30,36,42,48,60,72,84,96,108$ and 120 hours, according to the treatment. After this part, the ticks were taken to an incubator to finish the egg-laying and hatching. After 30 hours of water immersion, the teleogines had done the egg-laying,
\end{abstract}

\footnotetext{
${ }^{1}$ Médica Veterinária Autônoma. Universidade Federal de Santa Maria, UFSM, Santa Maria, RS. E-mail: carlini@portoweb.com.br

${ }^{2}$ Prof $^{\mathrm{a}} \mathrm{Dr}^{\mathrm{a}}$ do Dept $^{\mathrm{o}}$ de Medicina Veterinária Preventiva, Laboratório de Doenças Parasitárias, UFSM, Santa Maria, RS. E-mail: fefvogel@gmail.com

${ }^{3}$ Prof $^{\mathrm{a}} \mathrm{Dr}^{\mathrm{a}}$ do Dept $^{\mathrm{o}}$ de Microbiologia e Parasitologia, Laboratório de Parasitologia Veterinária. UFSM, Santa Maria, RS. E-mail: sgmonteiro@uol.com

${ }^{4}$ Prof. Dr. do Dept ${ }^{\circ}$ de Zootecnia, Faculdade de Agronomia Eliseu Maciel, Universidade Federal de Pelotas, UFPel, Pelotas, RS. E-mail: roll@hotmail.com

*Autor para correspondência
} 
however more them half of the eggs did not hatch. After 48h, half of the ticks had done the laying and the hatching rate was lower than $31 \%$. After $84 \mathrm{~h}$, there were no more hatching and the treatment had $100 \%$ efficacy. After analyzing the results, we can conclude that water immersion for more than 2 hours have a negative effect on cattle ticks reproduction parameters, through alterations in the egg-laying and in the hatching.

Key words: Distilled water, immersion, Rhipicephalus (Boophilus) microplus, posture outbreak

A infestação pelo carrapato Rhipicephalus (Boophilus) microplus é uma das doenças parasitárias de maior impacto econômico no rebanho bovino brasileiro. Segundo Grisi et al. (2002) estas perdas econômicas estão estimadas em valores acima de 2 bilhões de dólares ao ano, sendo que deste valor $75 \%$ são atribuídos aos carrapatos e o restante aos demais ectoparasitas, tais como miíase, mosca do chifre e piolhos.

As teleóginas têm alta eficiência reprodutiva completando seu ciclo de vida em aproximadamente 21 dias, conforme as condições ambientais. Visto que estas são capazes de ingerir entre 0,5 a $3 \mathrm{~mL}$ de sangue durante sua fase parasitária e que um bovino pode ser parasitado por milhares de carrapatos, perdas diárias de grande volume sanguíneo são inevitáveis. Além disto, este parasito pode causar baixa conversão alimentar, perda de peso crônica, redução da produção de leite, redução da qualidade do couro, lesões na pele que favorecem desenvolvimento de mí́ases, anemia e transmissão de patógenos tais como Babesia bigemina, B. bovis e Anaplasma marginale e transmissão de zoonoses.

Este ixodídeo é amplamente distribuído no território nacional, sendo reportado em pelo menos 95,6\% dos municípios brasileiros há bastante tempo (HORN, 1983). É um parasito hematófago preferencialmente de bovinos, podendo esporadicamente parasitar outros animais como equinos e ovinos (GONZALES, 2003). Esta espécie utiliza apenas um hospedeiro por ciclo, apresentando uma fase de vida livre que ocorre no solo e outra parasitária que ocorre no corpo do animal. Segundo Campbell e Glines (1979), além dos fatores ambientais, como temperatura ambiente e umidade relativa do ar, o alagamento do terreno exerce influência sobre o ciclo de vida dos ixodídeos.
Os carrapatos são parasitos de hospedeiros que vivem no solo utilizando oxigênio da atmosfera através de um sistema respiratório traqueal, com uma abertura ao meio exterior chamada de estigma respiratório (GONZALES, 2003). De um modo geral os carrapatos têm uma relação ambígua com a água, pois eles necessitam de elevada umidade relativa para manter o equilíbrio hídrico, mas parecem evitar o contato direto com ela (KRÖBER; GUERIN, 1999). No entanto, ainda guardam a capacidade de serem muito resistentes a imersão em água doce e água salgada (GONZALES, 2003). Considerando que algumas áreas de distribuição do $R$. microplus podem se tornar naturalmente alagadas conforme a época do ano, decidiu-se investigar a ação deletéria da imersão de teleóginas em água, em condições controladas de laboratório.

Para este experimento foram utilizadas 510 teleóginas de $R$. microplus coletadas de bovinos naturalmente infestados provenientes de uma propriedade rural em Santa Maria, Rio Grande do Sul (RS). No laboratório, as mesmas foram mesuradas, lavadas em água corrente, secas em papel toalha e separadas em dez tratamentos, com dez teleóginas em cada um, com pesos similares, seguindo a técnica descrita por Drummond et al. (1973).

As teleóginas foram imersas em $20 \mathrm{~mL}$ de água destilada por $0,2,4,6,12,18,24,30,36,42,48,60$, 72, 84, 96, 108 e 120 horas, conforme tratamento. Após a imersão, as teleóginas foram secas em papel toalha e fixadas através de uma fita adesiva em placas de Petri, previamente identificadas. A seguir foram levadas a estufa climatizada, regulada a temperatura de $27{ }^{\circ} \mathrm{C}$, umidade relativa superior a $80 \%$ por 14 dias. Após o período de postura, o 
total de ovos foi pesado e transferido para tubos de ensaio, vedados com algodão hidrófobo, sendo novamente encaminhados para a estufa, onde foram mantidos por 26 dias. Após o período de incubação dos ovos, realizou-se a leitura do percentual de eclodibilidade larval. A eficiência do tratamento foi calculada segundo a técnica de Drummond et al. (1973). Todos os tratamentos foram realizados em triplicata e os resultados foram obtidos através de suas médias. A análise estatística foi realizada através do programa Minitab 16, a um nível de significância de 5\%.
Os resultados referentes ao percentual de inibição da postura, eclosão das larvas e eficiência do tratamento são mostrados na Tabela 1. Na análise de regressão linear foi verificada associação significativa $(P<0,001)$ entre a inibição de postura (x) e período de imersão em água (y) expressa na seguinte equação $\mathrm{y}=-7,69304+0,86717 \mathrm{x}$ (Gráfico 1). Em outra análise de regressão linear foi verificada associação significativa $(\mathrm{P}<0,001)$ entre a eclosão de postura (x) e período de imersão em água (y) expressa na seguinte equação $\mathrm{y}=64,33585$ - 0,60373x (Gráfico 2).

Tabela 1. Efeito de períodos crescentes de imersão em água destilada sobre os percentuais de inibição de postura, eclosão da postura e eficiência do tratamento sobre teleóginas de Rhipicephalus (Boophilus) microplus. Santa Maria, RS, 2009.

\begin{tabular}{ccccc}
\hline TRATAMENTO & $\begin{array}{c}\text { PERÍODO DE } \\
\text { IMERSÃO } \\
\text { (horas) }\end{array}$ & $\begin{array}{c}\text { INIBIÇÃO DA } \\
\text { POSTURA } \\
(\%)\end{array}$ & $\begin{array}{c}\text { ECLOSÃO DA } \\
\text { POSTURA } \\
(\%)\end{array}$ & $\begin{array}{c}\text { EFICIÊNCIA DO } \\
\text { TRATAMENTO (\%) }\end{array}$ \\
\hline I & 0 & 0 & 98 & 0 \\
II & 2 & 6 & 55 & 66 \\
III & 4 & 0 & 83 & 18 \\
IV & 6 & 3 & 30 & 72 \\
V & 12 & 6 & 45 & 57 \\
VI & 18 & 0 & 17 & 88 \\
VII & 24 & 3 & 80 & 11 \\
VIII & 30 & 13 & 58 & 53 \\
IX & 36 & 33 & 55 & 67 \\
X & 42 & 40 & 5 & 97 \\
XI & 48 & 56 & 31 & 78 \\
XII & 60 & 40 & 45 & 55 \\
XIII & 72 & 53 & 30 & 86 \\
XIV & 84 & 86 & 0 & 100 \\
XV & 96 & 63 & 0 & 100 \\
XVI & 108 & 96 & 0 & 100 \\
XVII & 120 & 100 & 0 & 100 \\
\hline
\end{tabular}

Fonte: Elaboração dos autores. 
Gráfico 1. Análise de regressão linear com os parâmetros de inibição de postura (IP) (x) e período em imersão em água (H) (y) em teleóginas de R. microplus. Santa Maria, RS, 2009.

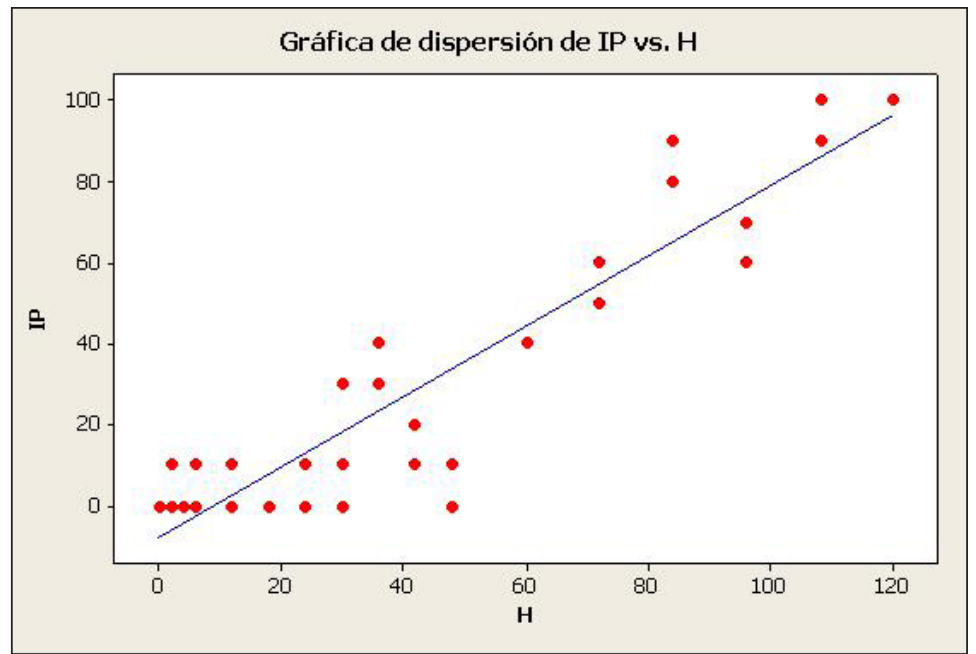

Fonte: Elaboração dos autores.

Gráfico 2. Análise de regressão linear com os parâmetros de eclosão da postura (EP) (x) e período em imersão em água (H) (y) em teleóginas de R. microplus. Santa Maria, RS, 2009.

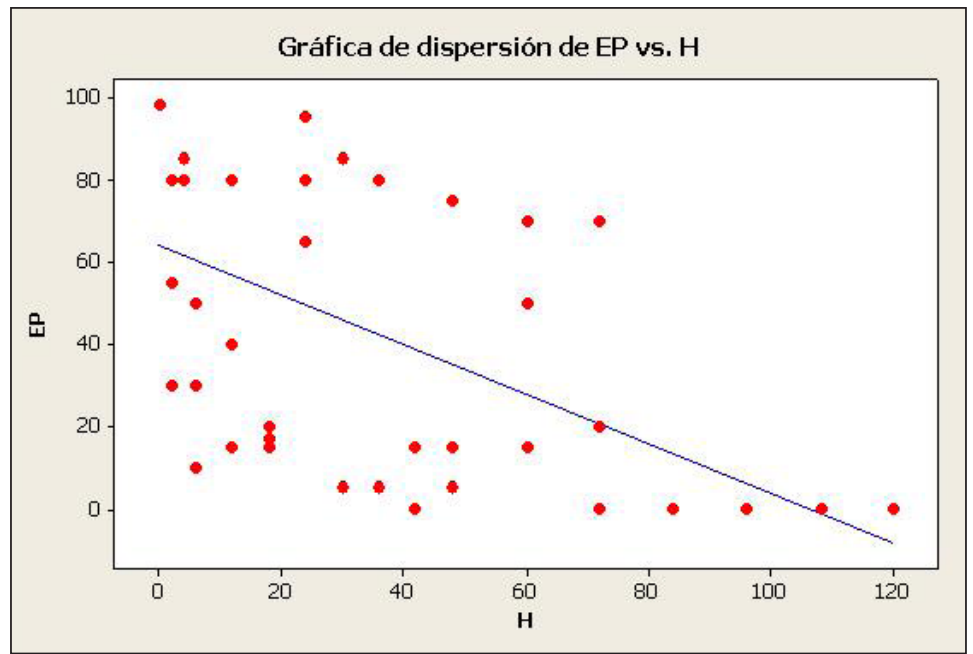

Fonte: Elaboração dos autores.

Experimentos relacionados à imersão de carrapatos em água ainda são escassos no Brasil e merecem mais atenção, uma vez que a maioria das pesquisas é realizada em outros países, dificultando a aplicação destes resultados na realidade brasileira devido à variação das condições ambientais conforme a região. Campos alagados atuam como uma forma de controle natural do carrapato bovino, uma vez que a imersão em água afeta negativamente a reprodução destes parasitos, reduzindo a produção e sobrevivência de seus ovos. Os testes estatísticos comprovaram um alto índice de associação entre o tempo que as teleóginas ficaram imersas em água com o aumento da inibição de postura e redução na taxa de eclosão larval.

A partir de $30 \mathrm{~h}$ de imersão das teleóginas a maioria dos ovos não eclodiram, sendo que estas realizaram postura dos ovos, porém este período 
de imersão alterou a capacidade de eclosão larval. Considerando que aproximadamente $50 \%$ das fêmeas não realizaram postura após 48h, este resultado sugere que períodos de imersão superiores a 2 dias seja um fator limitante na reprodução destes ectoparasitas. A eficiência do tratamento teve seu valor máximo a partir de $84 \mathrm{~h}$ de imersão, onde foi observado que $14 \%$ das teleóginas realizaram postura, porém estes ovos não eclodiram e consequentemente não se tornaram larvas. Após $120 \mathrm{~h}$ de imersão todas as fêmeas morreram sem realizar postura.

Verificou-se no presente experimento que somente $3 \%$ e $13 \%$ das teleóginas mantidas por $24 \mathrm{~h}$ e $30 \mathrm{~h}$ imersas não realizaram postura de ovos. Estes resultados são divergentes de Racioppi e Lombardero (1984) que observaram que as fêmeas mantidas durante $24 \mathrm{~h}$ em imersão, apenas três realizaram posturas e todas imersas por $30 \mathrm{~h}$ morreram sem colocar ovos.

Sutherst (1971) percebeu através de seus estudos que o peso total da massa de ovos já diminuiu significativamente a partir de $24 \mathrm{~h}$ de imersão, semelhante ao observado no presente experimento onde se observou redução da massa a partir de $30 \mathrm{~h}$, sendo isto foi atribuído principalmente a gradativa inibição da postura das teleóginas.

Concordando com os resultados obtidos neste trabalho, Louzada e Daemon (2003) verificaram uma queda na produção de ovos quando o tempo de imersão das teleóginas foi de $48 \mathrm{~h}$. No atual estudo cerca de $50 \%$ das fêmeas sobreviveram mesmo após $48 \mathrm{~h}$ de imersão e 44\% realizaram ovoposição, porém a eclosão larval foi baixa (31\%) elevando a eficiência do tratamento para $78 \%$.

Paula et al. (2004) em seus estudos com o Amblyomma cajennense chegaram a conclusão que larvas e ninfas alimentadas resistem menos à imersão do que as larvas não alimentadas. Cançado et al. (2006) estudando a imersão de larvas ingurgitadas de Amblyomma dubitatum verificaram que a partir de $72 \mathrm{~h}$ de imersão já havia efeito deletério significativo e que nenhuma destas sobreviveu após 8 semanas de imersão.

Paula et al. (2005) pesquisaram sobre o efeito da imersão em água de larvas e ninfas ingurgitadas de Amblyomma cajennense. Estes autores verificaram que nenhuma ninfa sobreviveu após 96h de imersão, o período de pré-ecdise aumentou conforme o tempo de imersão e que o percentual de ecdise ninfal reduziu conforme o período. No atual estudo, após $96 \mathrm{~h}$ de imersão houve sobrevivência de 38\% das fêmeas, no entanto não houve eclosão de suas posturas. Apesar do Amblyomma e do Rhipicephalus pertencerem a gêneros diferentes, pode-se observar que em ambos a imersão em água provoca efeito negativo na reprodução das teleóginas, no entanto o período de sobrevivência (em horas) foi divergente entre eles, indicando uma tolerância a água superior do Rhipicephalus.

Resultados diferentes foram constatados por Koch (1986) que encontrou $100 \%$ e $97 \%$ de sobrevivência de larvas ingurgitadas de $A$. americanum em um período de um e cinco dias de imersão, respectivamente. A sobrevivência das larvas chegou a $28 \%$ após seis dias e $0 \%$ após sete dias. No presente experimento nenhuma das teleóginas sobreviveu após cinco dias, sendo que este fato pode ser atribuído a menor resistência das teleóginas em relação às larvas ingurgitadas. Penna (1999) observou uma mortalidade progressiva e significativa das ninfas ingurgitadas de $R$. sanguineus a partir de $6 \mathrm{~h}$ de imersão. Com isto pode-se observar que a ninfa ingurgitada de $R$. sanguineus é mais sensível a imersão em água que as teleóginas de $R$. microplus, uma vez que após $6 \mathrm{~h}$ não houve morte das teleóginas.

A maioria das teleóginas realizou postura de ovos mesmo após longos períodos de imersão (até 108h), no entanto suas posturas foram 100\% inférteis a partir de $84 \mathrm{~h}$. Conclui-se que a imersão de $R$. microplus em água por períodos superiores á $2 \mathrm{~h}$ apresenta efeito deletério na postura de ovos e eclosão larval, significando uma redução na progênie destas teleóginas. 


\section{Referências}

CAMPBELL, A.; GLINES, M. V. Development, survival and oviposition of the rabbit tick, Haemaphysalis leporispalustris (Packard) (Acari: Ixodidae), at constant temperatures. Journal of Parasitology, Lawrence, v. 65, n. 5, p. 777-782, 1979.

CANÇADO, P. H. D.; CHANCÓN, S. C.; PIRANDA, E. M.; PAULA, A. R.; FACCINI, J. L. H. Efeito da imersão de larvas e ninfas ingurgitadas de A. Neumann, 1899 (Acari: Ixodidae) em água destilada. Revista Brasileira de Parasitologia Veterinária, Jaboticabal, v. 15, n. 1, p. 17-22, 2006.

DRUMMOND, R. O.; ERNST, S. E.; TREVINO, J. L.; GLADNEY, W. J.; GRAHAM, O. H. Boophilus annulatus and Boophilus microplus: laboratory tests for insecticides. Journal of Economic Entomology, Lanham, v. 66, p. 130-133, 1973.

GONZALES, J. C. O controle do carrapato do boi. 3. ed. Passo Fundo: Universidade de Passo Fundo, 2003. 128 p.

GRISI, L.; MASSARD, C. L.; MOYA BORJA, G. E.; PEREIRA, J. B. Impacto econômico das principais ectoparasitoses em bovinos no Brasil. A Hora Veterinária, Porto Alegre, v. 21, n. 125, p. 8-10, 2002.

HORN, S. C. Prováveis prejuizos causados pelos carrapatos. 2. ed. Brasília: Ministério da Agricultura. 1983. (Boletim de Defesa Sanitária Animal, n. especial).

$\mathrm{KOCH}, \mathrm{H}$. G. Survival of the lone star tick (Acari: Ixodidae) under flooding conditions: a laboratory evaluation. Journal of Economic Entomology, Lanham, v. 79, n. 6, p. 1555-1557, 1986.

KRÖBER, T.; GUERIN, P. M. Ixodid ticks avoid contact with liquid water. Journal of Experimental Biology, Cambrigde, v. 202, n. 14, p. 1877-1883, 1999.
LOUZADA, G. L.; DAEMON, E. Efeito da imersão de fêmeas ingurgitadas de Boophilus microplus (Canestrini, 1887) (Acari: Ixodidae) em água destilada sobre os parâmetros biológicos ligados à oviposição. Revista Brasileira de Parasitologia Veterinária, Jaboticabal, v. 12, n. 3, p. 115-120, 2003.

PAULA, A. R.; PIRANDA, E. M.; FACCINI, J. L. H.; DAEMON, E. Efeito da imersão de larvas e ninfas ingurgitadas de Amblyomma cajennense (Fabricius, 1787) (Acari: Ixodidae) em água destilada. Revista Brasileira de Parasitologia Veterinária, Jaboticabal, v. 14, n. 2, p. 65-69, 2005.

. Efeito da imersão de larvas não alimentadas de Anocentor nitens (Neumann, 1897) e de Amblyomma cajennense (Fabricius, 1787) (Acari: Ixodidae) em água destilada. Revista Brasileira de Parasitologia Veterinária, Jaboticabal, v. 13, n. 1, p. 13-17, 2004.

PENNA, A. P. Efeito da imersão em água destilada sobre as fases de vida livre do ciclo evolutivo de Rhipicephalus sanguineus (Latreille, 1806) (Acari: Ixodidae). 1999. Dissertação (Mestrado em Medicina Veterinária) - Universidade Federal Rural do Rio de Janeiro, Seropédica.

RACIOPPI, O.; LOMBARDERO, O. J. Comportamiento de los desoves y larvas de Boophilus microplus en inmersion. Buenos Aires. Veterinaria Argentina, v. 1, n. 16, p. 576-581, 1984.

SUTHERST, R. W. An experimental investigation into the effects of flooding on the ixodid tick Boophilus microplus (Canestrini). Oecologia, Berlim, n. 6, p. 208222, 1971. 